\title{
An Approach to Quantitative Estimation of Fragmentation of Molecular Ions in Mono-substituted Alkanes
}

\author{
ShIGERU IKUTA*, KenJi YoshiHARA* AND TAKanOBU SHIOKAWA*
}

(Received 6 March 1975)

\begin{abstract}
Calculation on the breakdown curves of molecular ions has been done applying molecular orbital treatment. In this treatment the following assumption is adopted: the scission probability of each bond is proportional to the partial bond population of the corresponding bond in the particular occupied MO which correlates to the particular ionization. The calculated breakdown curves were compared with those experimentally obtained after charge exchange with various positive ions. The fairly good agreement between them is obtained, if additional contributions of the processes of electron redistribution and secondary scission are considered. The degree of the transition from electronically excited molecular ion to the lower exited one was also estimated.
\end{abstract}

\section{Introduction}

We have already proposed the fragmentation mechanisms of molecular ions in monosubstituted alkanes based on the molecular orbital (MO) treatment. ${ }^{(-4)}$ According to these fragmentation mechanisms, mass spectra after electron impact or charge exchange with positive ions can be understood qualitatively in relation to an absorbed energy. In the lower energy region, the fragment ions directly produced from the molecular ion can be predicted using the MO assignments in various occupied molecular orbitals. And in the higher energy region, considerations on electron redistribution and secondary scission of the primary fragment ions should be needed.

In comparison with the quasi-equilibrium theory (QET) proposed by Rosenstock et al. ${ }^{5)}$ and the molecular orbital theory proposed by Hirota et al. ${ }^{6}$, the above proposal has the advantages which lead us to the better understanding of the fragmentation phenomena, but it also requires quantitative determination of the contribution of the mixed processes in the fragmentation concerned.

In this paper, therefore, we have further studied whether the quantitative estimation using our proposal is possible in explanation of the breakdown curve or not.

\section{Experimental and Calculation}

The breakdown curves (mass spectra as an absorbed energy) were obtained by charge exchange with various positive ions. The double mass spectrometer of a perpendicular type was used. The details of the apparatus were already shown elsewhere." The reaction chamber was kept below $6 \times 10^{-6}$ Torr to avoid the consecutive ion-molecule reactions.

The electronic structures of the molecule were calculated by the use of the Extended Hückel MO method, ${ }^{8)}$ and the calculation was performed at the Computer Center of Tohoku University.

\section{Results and Discussion}

- Department of Chemistry, Faculty of Science, Tohoku University, Aoba, Aramaki, Sendai 980 
Our fragmentation mechanisms already proposed are summarized as follows:" 1) The bond at which the electrons are localized on the corresponding occupied molecular orbital is firstly subjected to scission after the loss of the electron.

(2) Process (1) competes with the electron redistribution process, which is affected by the character of the molecule.

(3) In the higher energy region, secondary scission of the primary fragment ions occurs.

Based on the process (1), it is possible to predict which bond is to be subjected to scission, using the MO assignment in the corresponding occupied molecular orbital. For example, when the occupied MO consists of the lone pair electron not belonging to the chemical bond, the ionization from this orbital will produce the molecular ion, and when the MO consists of the $\mathrm{C}-\mathrm{X}$ bonding character $(\sigma(\mathrm{C}-\mathrm{X}))$, the ionization will result breaking of the $\mathrm{C}-\mathrm{X}$ bond. In this case, it is important to know which fragment will become to be a positive ion. It is already pointed out that the lower the ionization potential of the fragment is, the more easily it becomes a positive ion."

In Fig. 1 (A) the abundance of the $\mathrm{I}^{+}$ion produced after charge exchange of various $\mathrm{RI}$ $\left(\mathrm{R}=\mathrm{CH}_{3}, \mathrm{C}_{2} \mathrm{H}_{5}, \mathrm{n}-\mathrm{C}_{3} \mathrm{H}_{7}\right.$, and $\left.\mathrm{n}-\mathrm{C}_{4} \mathrm{H}_{9}\right)$ is shown as an absorbed energy, and in Fig. 1 (B) the abundance of the $\mathrm{X}^{+}$ion produced after charge exchange in various $\mathrm{CH}_{3} \mathrm{X}(\mathrm{X}=$ $\mathrm{Cl}, \mathrm{Br}$, and $\mathrm{I})$ is also shown. The ionization potential of the corresponding radical decreases

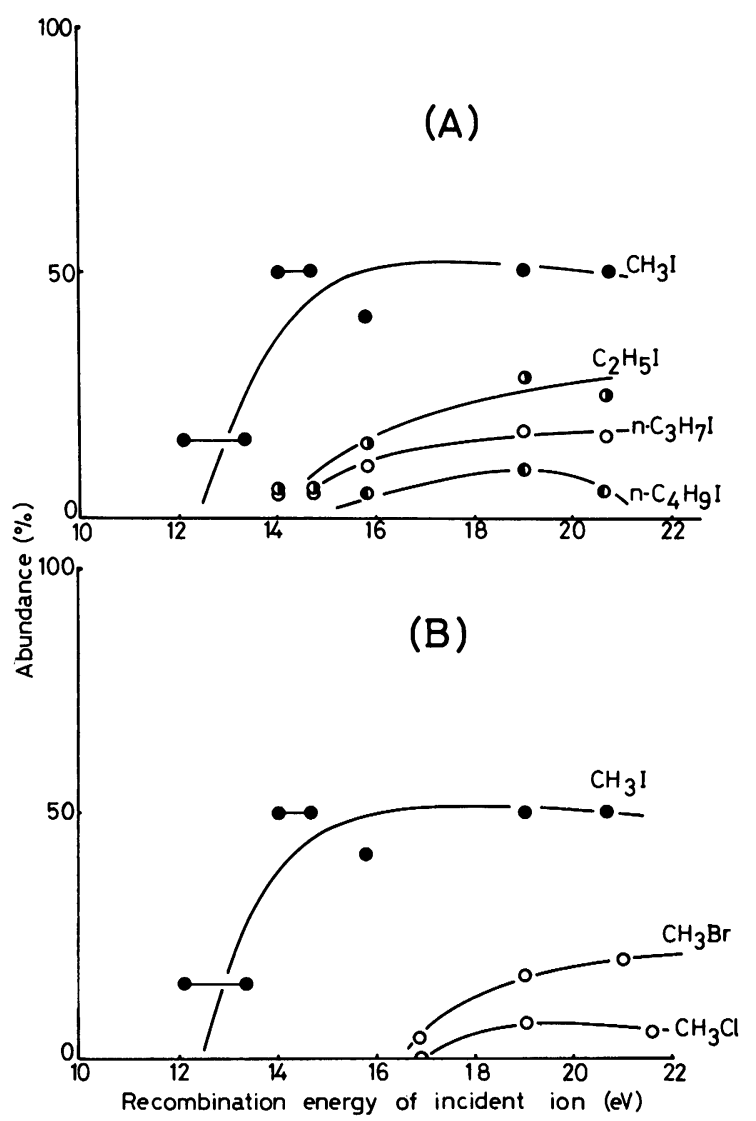

Fig. 1. Abundance of the $\mathrm{I}^{+}$ion produced after charge exchange of various RI (A) and that of the $\mathrm{X}^{+}$ion produced after charge exchange of various $\mathrm{CH}_{3} \mathrm{X}(\mathrm{B})$. 
as follows: I. P. $\left(\mathrm{CH}_{3}\right)>$ I. P. $\left(\mathrm{C}_{2} \mathrm{H}_{5}\right)>$ I. P. $\left(n-\mathrm{C}_{3} \mathrm{H}_{7}\right)>$ I. P. $\left(\mathrm{n}-\mathrm{C}_{4} \mathrm{H}_{9}\right)$; I. P. $(\mathrm{CI})>$ I. P. $(\mathrm{Br})>\mathrm{I}$. P. (I). According to these figures and to the order of the ionization potential, it is obvious that the lower the ionization potential of the fragment is, the more easily it becomes a positive ion.

In the case of the occupied MO consisting of various bonding characters, the scission probability of each bond can be estimated as follows: it is proportional to the partial bond population $\left(2 \mathrm{C}_{i} \mathrm{C}_{j} \mathrm{~S}_{i j}\right.$, where $\mathrm{C}_{i}$ and $\mathrm{C}_{j}$ are the eigenvectors of the $i$-th and $j$-th atomic orbitals, and $S_{i j}$ is the overlap integral between the $i$-th and $j$-th atomic orbitals) of the corresponding bond. Therefore, the fragment ions, which are produced by the primary scission of the molecular ion, will be predicted by the ratio of the partial bond population in the corresponding occupied MO.

In addition to the direct scission from the moleclar ion as stated above, we have to consider the second process, i. e. the electron redistribution process. It is generally found that the electronically excited molecular ion would change into the lower excited or ground ones before dissociation. Therefore, it is necessary to estimate the degree of transition

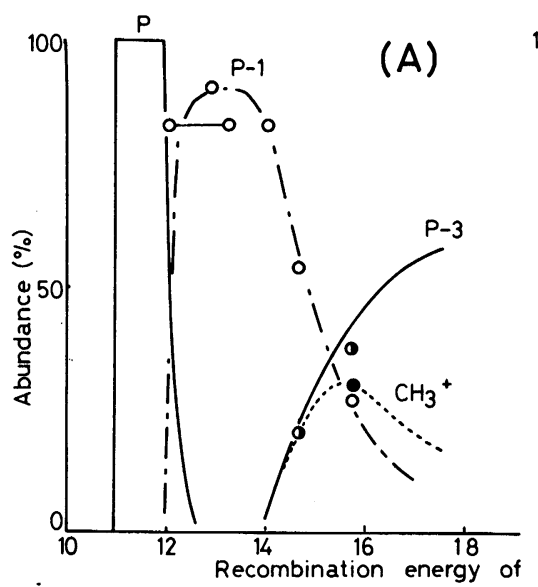

from the electronically excited molecular ion to the lower excited states. And further, in the higher energy region, consideration on the secondary scission (and those of the higher order) should be necessary. It is very important to investigate the above three processes precisely in quantitative interpretation of the actual breakdown curve of the molecular ion.

In this paper, the breakdown curve obtained after charge exchange is compared with that calculated based on our process (1), and the probability of the process (2) is estimated.

\subsection{Methanol $\left(\mathrm{CH}_{3} \mathrm{OH}\right)$}

The breakdown curve experimentally obtained after charge exchange in methanol ${ }^{10.11}$ and calculated one based on our process (1) are shown in Fig. 2 (A) and (B), respectively. The observed ionization potentials, ${ }^{(2)}$ calculated MO energies, and the values of the partial bond population in the corresponding $\mathrm{MO}$ are shown in Table 1. A good accordance between the appearance potentials of the fragment ions in the breakdown curve and those of the corresponding bands in the photoelectron spectra has been already pointed out." From these results the following conclusions are shown. As shown in Table 1, the highest occupied MO almost consists of the lone pair electron of the

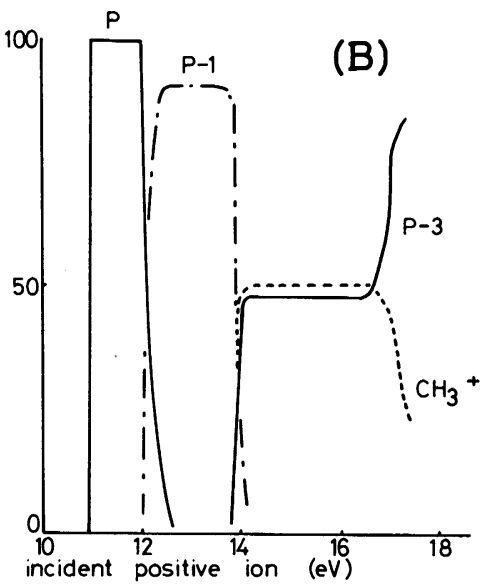

Fig. 2. Comparison of the observed breakdown curve $(A)^{10)}$ with calculated one (B) based on our process (1) in methanol. 
oxygen atom, and ionization from this orbital produces the molecular ion. The second occupied MO consists of the $\mathrm{C}-\mathrm{H}$ bonding character (and a little $\mathrm{O}-\mathrm{H}$ bonding character), and ionization produced the abundant $\mathrm{p}-1$ ion ( $p$ denotes the molecular ion). The third occupied MO consists of both $\mathrm{C}-\mathrm{H}$ bonding character ( $46 \%$ ) and $\mathrm{C}-\mathrm{O}$ bonding character (50\%). Therefore, the abundance of the $\mathrm{CH}_{3}{ }^{+}$ ion should be approximately equal to that of the hydrogen-deficient ion $(p-3)$. However, as shown in Fig. 2 (A), the maximum abundance of the $\mathrm{CH}_{3}{ }^{+}$ion only amounts to about $30 \%$. Therefore, 40 percents* of electronically excited molecular ions produced by the ionization of the third occupied MO can be assumed to change into the lower excited or ground ones before dissociation.

\subsection{Methylbromide $\left(\mathrm{CH}_{3} \mathrm{Br}\right)$}

The breakdown curve obtained after charge exchange and calculated one based on the process (1) in this work are shown in Fig. 3 (A) and (B), respectively. The good accordance between the appearance potentials of the fragment ions and those of bands in the photoelectron spectra has been already pointed out. ${ }^{2}$ As shown in the figure, the production of $\mathrm{CH}_{3} \mathrm{Br}^{+}$and $\mathrm{CH}_{3}^{+}$(and $\mathrm{Br}^{+}$) ions in the experimental curves is well correlated to that in calculated ones. Though the $\mathrm{CH}_{2} \mathrm{Br}^{+}$ion in the calculated breakdown curve amounts to $100 \%$, this ion only comprises about $30 \%$ in the observed one. Therefore, $70 \%$ of the electronically excited molecular ions produced by the ionization of the third occupied MO can be assumed to change into the lower

Table 1. Observed ionization potentials, ${ }^{12)}$ calculated ionization potentials, and ratio of the partial bond population in methanol.

\begin{tabular}{l|c|c|cc}
\hline Occupied MO & Obs. I. P. $(\mathrm{eV})^{(2)}$ & Calc. I. P. (eV) & \multicolumn{2}{|c}{$\begin{array}{c}\text { Ratio of partial } \\
\text { bond population (\%) }\end{array}$} \\
\hline Highest MO & 10.95 & 13.00 & $n(0) 88.4$ & $\sigma(\mathrm{CH}) 11.6$ \\
Second MO & 12.66 & 13.44 & $\sigma(\mathrm{CH}) 64.4$ & $\sigma(\mathrm{OH}) 30.6$ \\
Third MO & 15.09 & 14.81 & $\sigma(\mathrm{CO}) 50.8$ & $\sigma(\mathrm{CH}) 46.2$ \\
\hline
\end{tabular}
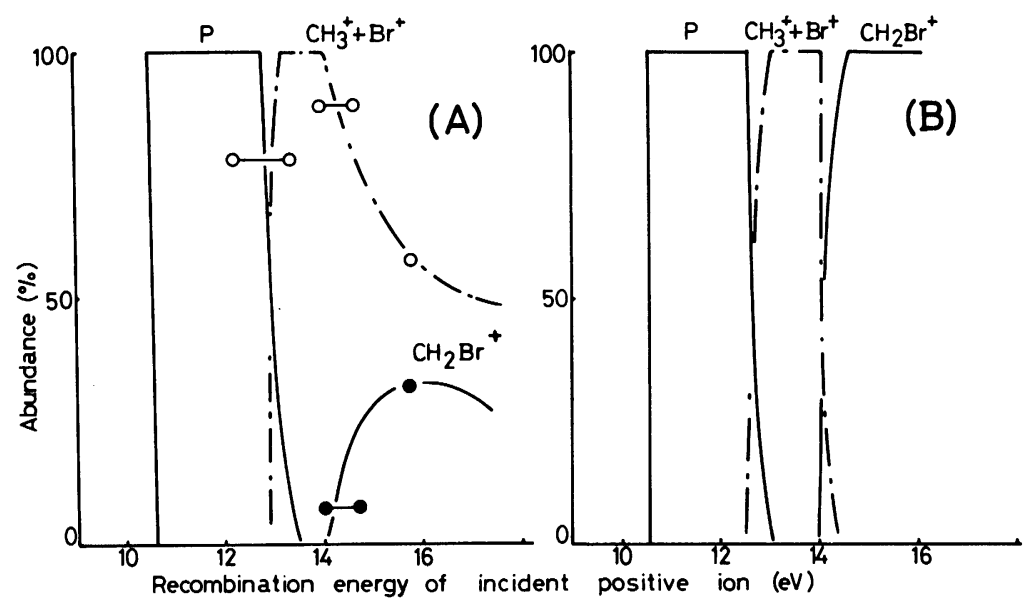

Fig. 3. Comparison of the observed breakdown curve (A) with calculated one (B) based on our process (1) in methylbromide.

$$
\frac{50-30}{50} \times 100 \%
$$




\section{An Approach to Quantitative Estimation of Fragmentation of Molecular Ions in Mono-substituted Alkanes}

excited or ground ones, and then decomposes to smaller ions.

\subsection{Ethanol $\left(\mathrm{C}_{2} \mathrm{H}_{5} \mathrm{OH}\right)$}

The breakdown curve obtained after charge exchange in ethanol ${ }^{13)}$ and that calculated on the basis of the process (1) in this work are shown in Fig. 4 (A) and (B), respectively. In Table 2, the calculated MO assignments and the calculated partial bond populations in various occupied MO's are shown. The good accordance between the appearance potentials of the fragment ions and those of the bands in the photoelectron spectra has been already pointed out. ${ }^{3)}$ Though the double maxima in the $\mathrm{p}-1$ ion and $\mathrm{CH}_{2} \mathrm{OH}^{+}$ion cannot be accounted for by QET and MOT, these are successfully calculated in this work qualitatively as shown in Fig. 4 (B). In Table 2, the highest occupied MO consists of the lone pair electron of the oxygen atom and the $\mathrm{C}-\mathrm{H}$ bonding character, and therefore the ionization of this orbital will produce the molecular ion (p) and the hydrogen-deficient ion $(p-1)$. As the second occupied MO calculated in this work consists of the $\mathrm{C}-\mathrm{C}$ bonding character (70 \%) and the $\mathrm{O}-\mathrm{H}$ bonding character (or $n(0)$ ), the ionization will produce the abundant $\mathrm{CH}_{2} \mathrm{OH}^{+}$ion and a smaller quantity of the hydrogen-deficient ion. The third MO consists of the $\mathrm{C}-\mathrm{H}$ bonding character and the lone pair electron of oxygen atom. Therefore, the ionization of this lone pair electron would result the electron redistribution to the state of the lower excited molecular ion. As shown in Fig. 4 (B), the calculated $\mathrm{C}_{2} \mathrm{H}_{5}{ }^{+}$ion comprises $50 \%$, and this fact shows that the molecular
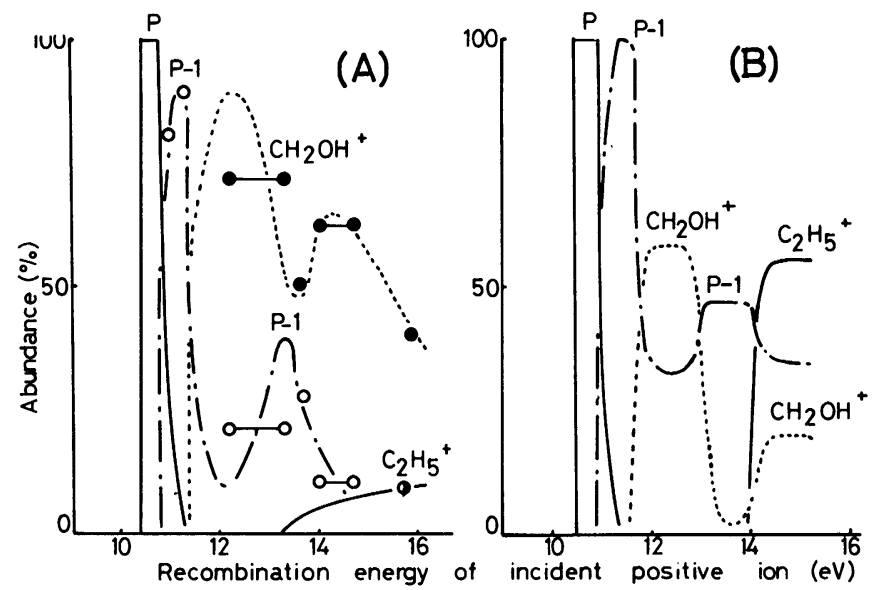

Fig. 4. Comparison of the observed breakdown curve (A) ${ }^{13)}$ with calculated one (B) based on our process (1) in ethanol.

Table 2. Calculated ionization potentials and ratio of the partial bond population in ethanol.

\begin{tabular}{|c|c|c|c|}
\hline Occupied MO & Calc. I. P. (eV) & \multicolumn{2}{|c|}{$\begin{array}{c}\text { Ratio of partial } \\
\text { bond population (\%) }\end{array}$} \\
\hline Highest MO & 12.81 & $n(0) 74.6$ & $\sigma(\mathrm{CH}) 25.4$ \\
\hline Second MO & 13.26 & $\sigma(\mathrm{CC}) 66.0$ & $\sigma(\mathrm{OH})($ or $n(0)) 34.0$ \\
\hline Third MO & 14.13 & $\sigma(\mathrm{CH}) 46.9$ & $n(0) 53.1$ \\
\hline Fourth MO & 14.22 & $\begin{array}{l}\sigma(\mathrm{CC}) 12.1 \\
\sigma(\mathrm{CH}) 33.9\end{array}$ & $\sigma(\mathrm{CO}) 54.0$ \\
\hline
\end{tabular}


ion produced by the ionization of the fourth occupied MO easily changes into the lower excited state.

Though there is a qualitative agreement between the observed and calculated breakdown curves, it is difficult to obtain a quantitative accordance by the above consideration of the process (1) alone. From these facts, it may be considered that the process (2) would play a very important role on the breakdown phenomena of the molecular ion.

\subsection{2-Propanethiol $\left(\mathrm{CH}_{3} \mathrm{CH}(\mathrm{SH}) \mathrm{CH}_{3}\right)$}

The breakdown curve obtained after charge exchange ${ }^{4}$ and that calculated on the basis of the process (1) in this work are shown in Fig. 5 (A) and (B). Though this molecule is relatively large, a good agreement is obtained in the low energy region. In our calculation the fourth occupied MO consists of the C-C bonding character $(52 \%)$ which would produce the $\mathrm{C}_{2} \mathrm{H}_{4} \mathrm{SH}^{+}$ion, and also consists of the $\mathrm{C}-$ $\mathrm{H}$ bonding character ( $48 \%$ ). It is considered that molecular ions produced by the ionization of $\mathrm{C}-\mathrm{H}$ bonding electron would easily change into the lower excited ones. Above $13 \mathrm{eV}$ the process (3) has to be considered in addition to the process (2). As shown in Fig. 5 (A), the secondary product $\mathrm{C}_{3} \mathrm{H}_{5}^{+}$(derived from $\mathrm{C}_{3} \mathrm{H}_{7}{ }^{+}$) is formed in the relatively low energy region. This has not been observed in the corresponding energy region for the smaller molecules discussed above.

These results and discussion lead us to the following conclusion.

(i) The observed breakdown curve is qualitatively in a good accordance with that calculated on the basis of the process (1).

(ii) The difference between the observed and calculated breakdown curves can be ascribed to the process (2) in the low energy region. (iii) In the higher energy region, the process (3) contributes to the fragmentation phenomena.

\section{References}

1) S. Ikuta, K. Yoshihara and T. Shiokawa, Mass Spectroscopy, 22, 233 (1974).

2) S. Ikuta, K. Yoshihara and T. Shiokawa, Bull. Chem. Soc. Japan, to be published.

3) S. Ikuta, K. Yoshihara and T. Shiokawa, Mass Spectroscopy, 22, 239 (1974)

4) K. Yoshihara, S. Ikuta, M. Kobayashi, and T. Shiokawa, Bull. Chem. Soc. Japan, to be published.

5) H. M. Rosenstock, M. B. Wallenstein, A. L. Wahrhaftig, and H. Eyring, Proc. Natl. Acad., U. S., 38, 667 (1952).

6) (a) K. Fueki and K. Hirota, Nippon Kagaku Zasshi, 81, $212(1960)$

(b) K. Hirota, Nippon Kagaku Zasshi, 91, 585 (1970).

7) T. Shiokawa, K. Yoshihara, M. Yagi, T. Omori, H. Kaji, M. Hiraga, T. Nagatani, and Y. Takita, Mass Spectroscopy, 18, $1230(1970)$
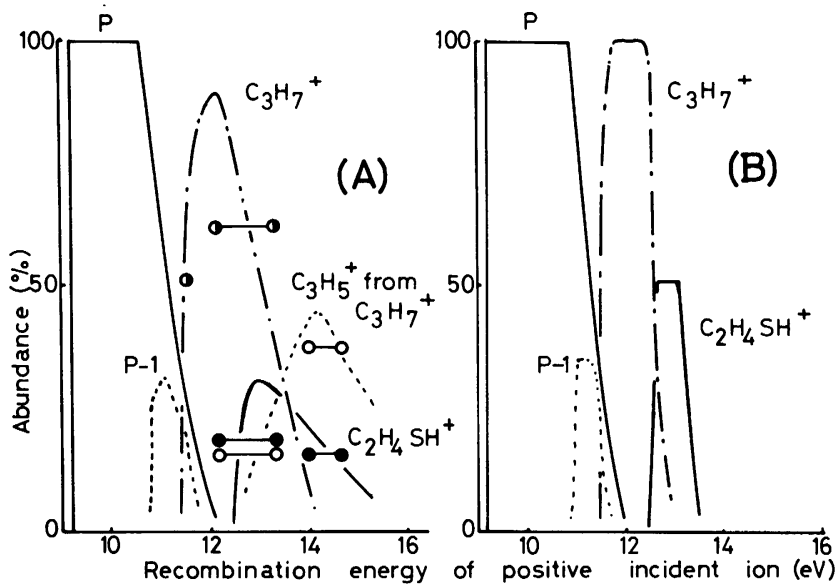

Fig. 5. Comparison of the observed breakdown curve (A) with calculated one (B) based on our process (1) in 2-propanethiol. 


\section{An Approach to Quantitative Estimation of Fragmentation}

of Molecular Ions in Mono-substituted Alkanes

8) R. Hoffmann, J. Chem. Phys., 39, 1397 (1963).

9) D. Stevenson, Discuss. Faraday Soc., 48, 581 (1952).

10) H. Sjögren, Arkiv Fysik, 31, 159 (1966).

11) T. Nagatani, K. Yoshihara, and T. Shiokawa, Bull. Chem. Soc. Japan, 46, 1306 (1973).
12) H. Ogata, H. Onizuka, Y. Nihei. H. Kamada, Bull. Chem. Soc. Japan, 46, 3036 (1973).

13) H. Koch and E. Lindholm, Arkir Fysik, 19, 123 (1961). 